\title{
Erratum to: Subjective Sleep Quality During Average Volume Assured Pressure Support (AVAPS) Ventilation in Patients with Hypercapnic COPD: A Physiological Pilot Study
}

Ernesto Crisafulli • Giuseppe Manni •

Marika Kidonias • Ludovico Trianni •

Enrico M. Clini

Published online: 30 October 2009

(C) Springer Science+Business Media, LLC 2009

Erratum to: LUNG (2009) 187(5): 299-305

DOI: 10.1007/s00408-009-9167-1

The corrected Table 2 now includes new $P$ values for VAS and Sleep Efficiency which are no longer significant when comparing PS with AVAPS.

The online version of the original article can be found under doi:10.1007/s00408-009-9167-1.

E. Crisafulli · G. Manni - M. Kidonias - L. Trianni - E. M. Clini Division of Pulmonary Rehabilitation, Ospedale Villa Pineta, Pavullo (MO), Italy

E. M. Clini

Department of Oncology, Haematology and Pneumology,

University of Modena and Reggio Emilia, Modena, Italy

E. M. Clini ( $\square)$

Division of Pulmonary Rehabilitation, University of Modena and Ospedale Villa Pineta, Via Gaiato 127, 41026 Pavullo (MO), Italy

e-mail: enrico.clini@unimore.it 
Table 2 Time course of the study outcomes recorded during both treatment periods

\begin{tabular}{|c|c|c|c|c|c|c|c|c|c|}
\hline \multirow[t]{3}{*}{ Variables } & \multicolumn{9}{|c|}{ PS vs AVAPS modality } \\
\hline & \multicolumn{4}{|l|}{ PS modality } & \multicolumn{4}{|c|}{ AVAPS modality } & \multirow[b]{2}{*}{$P^{* *}$} \\
\hline & T0 & $\mathrm{T} 1$ & $\mathrm{~T} 2$ & $P^{*}$ & T0 & $\mathrm{T} 1$ & $\mathrm{~T} 2$ & $P^{*}$ & \\
\hline $\mathrm{pH}$ & $7.37(0.02)$ & $7.37(0.02)$ & $7.38(0.02)$ & 0.872 & $7.37(0.03)$ & $7.38(0.01)$ & $7.39(0.01)$ & 0.296 & 0.175 \\
\hline $\mathrm{PaCO}_{2}(\mathrm{kPa})$ & $8.41(0.8)$ & $7.86(1.1)$ & $7.26(1.0)$ & 0.019 & $7.95(1.0)$ & $7.38(0.8)$ & $7.29(1.0)$ & 0.047 & 0.134 \\
\hline $\mathrm{PaO}_{2}(\mathrm{kPa})$ & $9.67(1.9)$ & $9.71(1.2)$ & $10.6(1.0)$ & 0.074 & $9.34(1.2)$ & $9.62(1.1)$ & $9.33(1.0)$ & 0.666 & 0.064 \\
\hline $\mathrm{PaO}_{2} / \mathrm{FiO}_{2}$ & $271.1(65.1)$ & $274.9(60.8)$ & $299.6(46.3)$ & 0.087 & $262.5(50.6)$ & $270.5(49.4)$ & $261.6(39.7)$ & 0.618 & 0.057 \\
\hline VAS (comfort to NPPV) (mm) & $57.5(31.8)$ & $53.2(26.2)$ & $49.5(26.3)$ & 0.019 & $49.5(24.1)$ & $46.2(23.6)$ & $43.0(20.5)$ & 0.075 & 0.056 \\
\hline Sleep quality questionnaire (score) & $5.1(1.7)$ & $5.1(1.6)$ & $4.7(1.3)$ & 0.219 & $5.1(2.0)$ & $5.0(2.1)$ & $4.1(2.2)$ & 0.001 & 0.531 \\
\hline
\end{tabular}

Values are given as mean (SD)

* Level of significance among times

** Level of significance between groups

Legend: for abbreviations see legend to Table 1 in original article 